\title{
Essai randomisé mesurant l'efficacité des modifications apportées au tableau de la valeur nutritive sur la compréhension et l'utilisation de l'information nutritionnelle par les adolescents et les jeunes adultes au Canada
}

\author{
E. Hobin, Ph. D. (1); J. Sacco, Ph. D. (1); L. Vanderlee, B. Sc. (2); C. M. White, M. Sc. (2); F. Zuo, M.H.P. (1); \\ J. Sheeshka, Ph. D. (3); G. McVey, Ph. D. (4); M. Fodor O’Brien, M. Sc. S. (1); D. Hammond, Ph. D. (2)
}

Cet article a fait l'objet d'une évaluation par les pairs.

Diffuser cet article sur Twitter

\section{Résumé}

Introduction : Compte tenu des changements proposés à l'étiquetage nutritionnel au Canada et de la rareté des travaux de recherche portant sur la compréhension et l'utilisation des tableaux de la valeur nutritive (tVN) chez les adolescents et les jeunes adultes, notre objectif consistait à réaliser un essai expérimental pour déterminer si les modifications apportées au tVN permettaient d'améliorer efficacement la façon dont les jeunes Canadiens interprètent, comparent et manipulent, sur le plan mathématique, l'information nutritionnelle figurant dans le tVN de denrées préemballées.

Méthodologie : Une enquête en ligne a été menée auprès d'un échantillon de consommateurs composé de 2010 Canadiens âgés de 16 à 24 ans. Nous avons réparti les participants de façon aléatoire en six groupes d'étude, et nous avons présenté à chacun des groupes deux des six tVN définis comme conditions expérimentales, selon un plan factoriel $2 \times 3$ : portion de référence ( $\mathrm{tVN}$ actuel et portions de référence normalisées pour tous les produits similaires) $\times$ pourcentage de la valeur quotidienne (\% VQ) (tVN actuel, ajout des descripteurs « faible/moyen/élevé » et ajout d'un code de couleurs). L'enquête comprenait sept tâches consistant à interpréter, comparer et manipuler, sur le plan mathématique, l'information nutritionnelle figurant dans les tVN. Des modèles de régression de Poisson modifiés ont été élaborés pour chacun des trois résultats.

Résultats : La capacité à comparer deux produits similaires s'est révélée significativement meilleure quand le tVN incluait une portion de référence normalisée ( $p \leq 0,001$ dans tous les cas). L'ajout de descripteurs ou d'un code de couleurs indiquant, sur le tVN, le \% VQ pour les calories et les nutriments a amélioré de façon significative la capacité des participants à interpréter correctement l'information à propos de ce $\%$ VQ ( $p \leq 0,001$ dans tous les cas). Le fait de présenter aux participants des portions de référence normalisées et des descripteurs du \% VQ a eu un effet modeste sur leur capacité à manipuler, sur le plan mathématique, l’information nutritionnelle pour calculer la valeur nutritive de plusieurs portions d'un produit (ratio relatif $=1,19$; intervalle de confiance à $95 \%: 1,04$ à 1,37 )

Conclusion : La normalisation des portions de référence et l'ajout d'information sur l'interprétation du \% VQ dans le tVN ont permis aux jeunes Canadiens de mieux comprendre et utiliser l'information nutritionnelle. Il faut néanmoins faire preuve de prudence avant de généraliser les résultats de l'enquête à l'ensemble des jeunes Canadiens en raison de l'échantillonnage de la population à l'étude. D'autres travaux de recherche sont nécessaires afin de reproduire cette étude au Canada avec un échantillon plus hétérogène, et en utilisant un éventail de produits alimentaires et de catégories d'aliments.

Mots-clés : adolescents, jeunes adultes, politique nutritionnelle, étiquetage des aliments
Principales constatations

- Notre étude fournit des données probantes préliminaires, jusqu'à présent inexistantes au Canada, à l'appui de l'efficacité des modifications apportées au tableau de la valeur nutritive (tVN) pour permettre aux consommateurs de comprendre et d'utiliser l'information nutritionnelle.

- Les résultats laissent penser que la normalisation des portions de référence et l'utilisation de descripteurs ou d'un code de couleurs pour interpréter le pourcentage de valeur quotidienne (\% VQ) dans le tVN aident les jeunes Canadiens à interpréter, à comparer et à manipuler, sur le plan mathématique, l'information nutritionnelle. Il faut néanmoins faire preuve de prudence avant de généraliser les résultats de l'enquête à l'ensemble des jeunes Canadiens en raison de l'échantillonnage de la population à l'étude.

- Les résultats peuvent être utilisés pour étayer l'analyse en cours sur les changements proposés au tVN.

\section{Introduction}

La mauvaise alimentation figure parmi les principaux facteurs de risque de maladie chronique et de décès prématuré au Canada $^{1}$. Un apport très élevé en calories, en gras saturés et en sodium est associé à

Rattachement des auteurs :

1. Santé publique Ontario, Toronto (Ontario), Canada

2. Université de Waterloo, Waterloo (Ontario), Canada

3. Université de Victoria, Melbourne, Australie

4. Hospital for Sick Children, Toronto (Ontario), Canada

Correspondance : Erin Hobin, Santé publique Ontario, 480 University Avenue, suite 300, Toronto (Ontario) M5G 1V2; tél. : 647-260-7198; courriel : Erin.Hobin@0ahpp.ca 
un risque accru d’obésité, de diabète sucré et de maladies cardiaques ${ }^{2-4}$. Les maladies d'origine nutritionnelle, comme l'obésité et le diabète, sont de plus en plus courantes chez les adolescents et les jeunes adultes, au Canada comme à l'échelle internationale ${ }^{5-8}$. L'adolescence et le début de l'âge adulte sont des stades dynamiques dans le développement humain associés à une plus grande autonomie, à des responsabilités accrues en matière d'achat et de préparation des aliments ainsi qu'à l'adoption d'habitudes alimentaires susceptibles d'être maintenues à long terme et de demeurer relativement stables tout au long de la vie ${ }^{9-11}$. C'est pourquoi des interventions axées sur la population dans le domaine de la nutrition devraient aider à l'adoption de saines habitudes chez les jeunes au Canada.

L'un des moyens de favoriser des choix alimentaires plus sains et éclairés consiste à fournir une information nutritionnelle claire et exacte. L'étiquetage nutritionnel obligatoire sur les aliments préemballés a été mis en œuvre en 2005 au Canada afin que les consommateurs puissent comparer les valeurs nutritionnelles des aliments et faire des choix éclairés ${ }^{12}$. En vertu de cette réglementation, le tableau de la valeur nutritive (tVN) doit figurer sur l'étiquette de la plupart des aliments préemballés. Le tVN renseigne, pour une portion, sur le nombre de calories et sur la teneur en 13 nutriments ainsi que sur le pourcentage associé à chaque élément en fonction des recommandations en matière d'apports nutritionnels pour un adulte consommant 2000 calories par jour (pourcentage de la valeur quotidienne [\% VQ]).

Le tVN représente la principale source d'information nutritionnelle au Canada : davantage de Canadiens déclarent utiliser l'information nutritionnelle figurant sur l'étiquette des produits préemballés, comparativement à toute autre source, devant Internet, les diététiciens et les médias de masse $^{13}$. De plus, les consommateurs canadiens ont indiqué préférer le tVN aux autres systèmes d'étiquetage nutritionnel des emballages lors d'une évaluation fondée sur les critères suivants : appréciation, caractère utile, crédibilité et influence sur les décisions d'achat ${ }^{14}$. Ces résultats sont conformes à ceux d'une grande quantité de travaux de recherche à l'échelle internationale selon lesquels les étiquettes obligatoires figurant sur les produits alimentaires ont une vaste portée et constituent des outils éducatifs valables et durables en matière de santé ${ }^{15}$.

Bien que le tVN soit largement utilisé, des recherches récentes ont mis en évidence diverses limites touchant sa compréhension et son utilisation par la population canadienne adulte ${ }^{16}$. D'abord, même si la majorité des adultes au Canada affirment que l'information fournie par le tVN est importante, ils trouvent difficile de comparer l'information nutritionnelle d'aliments similaires lorsque les portions de référence figurant dans le $\mathrm{tVN}$ des produits ne sont pas les mêmes. Bien que l’Agence canadienne d'inspection des aliments définisse des intervalles précis pour l'établissement de la portion de référence (dites « portions déterminées ») des différentes catégories d'aliments, c'est en définitive aux fabricants de produits alimentaires d'établi $r$ la portion de référence qui figurera dans le $\mathrm{tVN}^{17}$. Étant donné que les informations sur la valeur nutritive indiquées dans le tVN sont basées sur la portion de référence, l'utilisation d'une portion de référence uniformisée serait susceptible d'aider les consommateurs à comparer les teneurs en nutriments d'aliments similaires.

En outre, la plupart des adultes au Canada ne réussissent pas à comprendre ou à utiliser le \% VQ figurant dans le $\mathrm{tVN}^{16}$. Alors que ce \% VQ a pour objet de faciliter les comparaisons entre différents aliments et d'aider les consommateurs à déterminer si un aliment a une faible teneur ou une teneur élevée en un nutriment donné ${ }^{18}$, près du tiers des adultes canadiens ne comprennent pas qu'il peut les aider à comparer des aliments, et $74 \%$ sont incapables de l'interpréter pour déterminer si l'apport en un nutriment donné d'un aliment est élevé ou faible ${ }^{16}$. D’après certaines études $^{15,19}$, l'ajout de descripteurs simples (« faible », « moyen » ou " élevé ») ou d'un code de couleurs indiquant le \% VQ dans le tVN pourrait contribuer à améliorer la compréhension et par conséquent l'utilisation de l'information nutritionnelle. On prend également en considération ce type de formats de présentation de l'information nutritionnelle pour en faciliter l'interprétation dans des travaux d'examen des systèmes d'étiquetage nutritionnel des emballages ${ }^{20}$.

À notre connaissance, il n'existe au Canada aucune étude publiée portant sur la compréhension et l'utilisation du tVN des aliments préemballés chez les adolescents et les jeunes adultes. Les rares études menées auprès des adolescents à l'échelle internationale laissent penser que la compréhension et l'utilisation des étiquettes nutritionnelles au sein de ce groupe est faible ${ }^{19,21}$. La révision de la réglementation en matière d'étiquetage nutritionnel actuellement engagée au Canada constitue une occasion d'élaborer des exigences d'étiquetage permettant aux consommateurs de faire des choix plus sains en matière d'alimentation ${ }^{22}$. L'objectif de notre étude consistait à comparer, au moyen d'un essai expérimental, l'efficacité de $\mathrm{tVN}$ modifiés par rapport au tVN actuel en ce qui concerne la compréhension et l'utilisation de l'information nutritionnelle chez les adolescents et les jeunes adultes. $\mathrm{Au}$ nombre des modifications du tVN mises à l'essai figuraient la normalisation des portions de référence et l'ajout aux $\%$ VQ d'information visant à faciliter l'interprétation (descripteurs simples ou code de couleurs). Ces modifications ont été sélectionnées parce que l'affichage dans le $\mathrm{tVN}$ de portions de référence inégales d'un produit à l'autre et les difficultés d'interprétation du \% VQ ont été reconnus comme des obstacles importants à la compréhension et à l'utilisation du tVN au sein de la population adulte canadienne ${ }^{16}$. Plus particulièrement, nous avons examiné les répercussions de ces modifications du tVN sur la capacité des participants à interpréter, à comparer et à manipuler, sur le plan mathématique, l'information nutritionnelle figurant sur les produits préemballés.

\section{Méthodologie}

\section{Participants et recrutement}

En août 2014, une enquête en ligne a été menée à l'échelle du Canada auprès de 2010 participants âgés de 16 à 24 ans. Les 
participants ont été recrutés parmi les membres d'un panel national de consommateurs en ligne établi par Nielson, une entreprise spécialisée dans les études de marché (Nielsen : http://www.nielsen. com/ca/fr.html). Les membres du panel ont été recrutés au moyen de publicités en ligne et grâce aux médias sociaux, à des courriels ciblés, à des offres d'inscription conjointe et à du recrutement téléphonique. Dans le cadre de notre étude, un échantillon aléatoire de panélistes de Nielsen a reçu par courriel une invitation à remplir un questionnaire d'enquête. On a recruté un nombre égal d'hommes et de femmes ainsi qu'un nombre égal d'adolescents (16 à 18 ans) et de jeunes adultes (19 à 24 ans). Les participants résidant dans les territoires ont été exclus du cadre d'échantillonnage.

Les participants ayant rempli le questionnaire d'enquête ont reçu une rémunération d'environ 2 \$ à 3 \$. Les questionnaires étaient disponibles en anglais seulement, et tous les participants ont donné leur consententement. Le Bureau de l'éthique en recherche de l'Université de Waterloo a donné son approbation à cette étude.

\section{Plan d'étude}

Nous avons utilisé un plan expérimental faisant appel à la comparaison de groupes pour évaluer la compréhension et l'utilisation des formats modifiés du tVN par rapport à ceux du tVN actuellement en vigueur au Canada. Nous avons réparti les participants de façon aléatoire entre les groupes, chacun obéissant à l'une des six conditions d'étiquetage à l'étude (figure 1), selon un plan factoriel $2 \times 3$ : portion de référence (tVN actuel ou portions de référence normalisées pour tous les produits similaires) $\times$ pourcentage de la valeur quoti dienne (\% VQ) (tVN actuel, descripteurs «faible/moyen/élevé » ou code de couleurs). Chacun des groupes devait examiner simultanément les images illustrant deux marques fictives d'emballage de craquelins modifié de façon systématique. Les portions de référence normalisées ont été établies en fonction des recommandations du Guide alimentaire canadien. La catégorisation du \% VN répondait aux critères des documents éducatifs en ligne ${ }^{18}$ selon lesquels un apport de $5 \%$ VQ ou moins en un nutriment correspond à un «faible " apport ou au code vert, un apport de $6 \%$ à $14 \%$ VQ en un nutriment correspond à un apport « moyen » ou au code jaune et un apport de $15 \%$ VQ ou plus en un nutriment correspond à un apport « élevé » ou au code rouge. Bien que la normalisation des portions de référence ait une incidence sur tous les nutriments figurant dans le tVN, les outils additionnels d'interprétation (descripteurs simples ou code de couleurs) ont été appliqués uniquement aux calories et aux nutriments dits « négatifs » (c'est-à-dire les lipides totaux, les gras saturés et le sodium), car les consommateurs consultent le tVN pour vérifier la teneur en nutriments négatifs plus fréquemment que pour les nutriments positifs $^{23}$, et parce qu'il existe des données probantes plus solides à l'appui des associations entre les nutriments négatifs et le risque accru de maladie ${ }^{20}$.

Les valeurs nutritionnelles figurant dans le tVN étaient similaires à celles trouvées sur des emballages de craquelins du commerce, mais elles ont été modifiées de façon à ce qu'une option renferme un apport élevé (15\% ou plus de la VQ) ou modéré ( $6 \%$ à $14 \%$ de la VQ) en sodium, et que l'autre option renferme un faible apport ( $5 \%$ ou moins de la VQ) en sodium par portion, d'après les valeurs nutritionnelles de référence selon lesquelles $1500 \mathrm{mg} /$ jour est considéré comme un apport suffisant ${ }^{24}$. Les apports en sodium utilisés dans les six conditions expérimentales ont été équilibrés de manière à ce que, pour la moitié des participants, ce soit la première boîte de craquelins qui corresponde au produit ayant une faible teneur en sodium et que, pour l'autre moitié des participants, ce soit la seconde.

Les craquelins ont été choisis pour cette étude parce qu'il s'agit d'une collation très répandue qui plaît à un large éventail de consommateurs et que, du point de vue nutritif, c'est un choix considéré ni comme très bon ni comme très mauvais pour la santé. Les tVN paraissaient sur des images de boîtes de craquelins portant des noms commerciaux fictifs et présentant un poids uniforme de $225 \mathrm{~g}$. Les boîtes de craquelins étaient affichées à l'écran sous forme d'images en deux dimensions avec des vues du devant et du côté de l'emballage, pour permettre aux participants de voir l'information sur le produit, notamment la marque, le poids du produit et le tVN. Les boîtes demeuraient affichées à l'écran jusqu'à ce que le participant ait terminé de répondre au questionnaire.

\section{Mesures relatives à l'enquête}

Caractéristiques sociodémographiques et comportements à l'égard de la nutrition

Nous avons sélectionné des variables sociodémographiques (sexe, âge, région, niveau de scolarité " secondaire ou moins ", « collégial ou études universitaires inachevées " ou " diplôme universitaire »), la situation vis-à-vis de l'emploi et l'origine ethnique. En outre, nous avons demandé aux participants d'évaluer la qualité de leur alimentation et de préciser leur objectif relatif à leur poids ainsi que leurs responsabilités en matière d'achat et de préparation des aliments.

Pour caractériser les objectifs personnels relatifs au poids, nous avons demandé aux participants s'ils essayaient de perdre du poids, de prendre du poids, de maintenir leur poids ou s'ils n'avaient pas d'objectif relativement à leur poids. Cette question du sondage a été inspirée de l'étude National Health and Nutrition Examination Survey (NHANES) dans laquelle on demandait aux participants s'ils aimeraient avoir un poids supérieur ou inférieur à leur poids actuel ou s'ils aimeraient maintenir leur poids actuel ${ }^{25}$.

Nous avons enquêté sur les responsabilités des participants en matière d'achat et de préparation des aliments en leur demandant de préciser leur rôle selon les catégories suivantes : « principal responsable de l'achat des aliments », « principal responsable de la préparation des repas », « principal responsable de l'achat des aliments et de la préparation des repas » et "n'assume pas ces responsabilités ».

Enfin, comme dans d'autres études, nous avons évalué les connaissances des participants en ce qui concerne l'apport 
FIGURE 1

Six conditions associées au tableau de la valeur nutritive

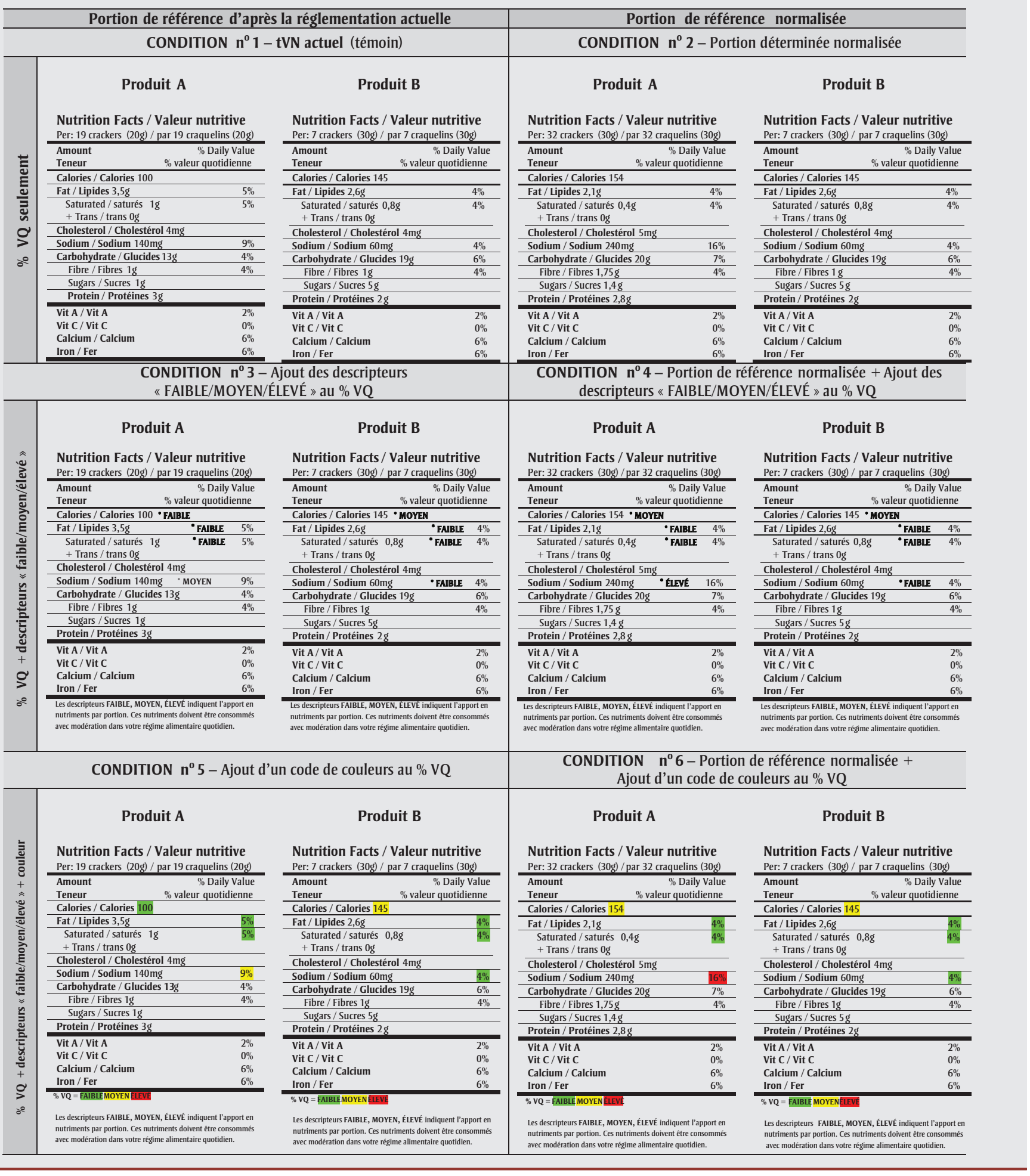

calorique recommandé, en leur demandant : « en moyenne, combien de calories est-il recommandé de consommer chaque jour pour un adulte [homme/femme] en santé qui a un niveau d'activité physique modéré et qui souhaite conserver un poids santé? ${ }^{26-28}$. Les réponses numériques (entre 0 et 100 000) ont été jugées correctes si elles rentraient dans l'intervalle de 1500 à 3000 calories par jour (d'après les recommandations de Santé Canada en ce qui concerne les besoins énergétiques quotidiens des jeunes adultes en fonction de divers niveaux d'activité physique) ${ }^{29}$. 


\section{Mesure des résultats}

Nous avons utilisé un questionnaire d'enquête en ligne pour évaluer la capacité des participants à interpréter, à comparer et à manipuler, sur le plan mathématique, l'information nutritionnelle présente dans le tVN. L'enquête comprenait sept tâches exigeant des participants qu'ils comprennent et qu'ils utilisent l'information du tVN paraissant sur les emballages de produits. Les sept tâches ont été élaborées à l'aide d'un outil employé dans une étude commandée par Santé Canada ${ }^{16}$ et réalisée par Mackison et ses collaborateurs ${ }^{30}$. Cet outil a permis de vérifier la validité et la fiabilité des tâches effectuées pour évaluer dans quelle mesure les consommateurs comprenaient et utilisaient l'information nutritionnelle figurant sur les étiquettes des produits et quelles étaient leurs perceptions à cet égard.

Interprétation de l'information sur le \% VQ dans les tVN

Nous avons utilisé deux tâches pour évaluer la capacité des participants à interpréter l'information sur le \% VQ. Nous avons d'abord demandé aux participants d'examiner une boîte de craquelins et de répondre à la question : « Ce produit contient-il une quantité de sodium élevée, modérée ou faible? » Nous leur avons ensuite demandé : « La quantité totale de lipides contenue dans une portion de ce produit est-elle élevée, modérée ou faible? »

\section{Comparaison de l'information figurant dans} les deux tVN

Nous avons utilisé trois tâches pour évaluer la capacité des participants à comparer l'information nutritionnelle figurant dans les deux tVN. Nous avons d'abord demandé aux participants de comparer les produits A et B et leur avons posé la question : «Quel produit est le meilleur choix pour une personne qui souhaite réduire son risque d'hypertension en diminuant sa consommation de sodium? » Nous leur avons ensuite demandé : « Quel produit est le meilleur choix pour une personne qui souhaite consommer moins de calories? » Enfin, nous leur avons demandé : « Ces deux produits ont-ils des teneurs en sodium comparables? » Les choix de réponse étaient « les deux ont une teneur élevée en sodium », « les deux ont une faible teneur en sodium », " le produit A a une faible teneur en sodium et le produit B a une teneur élevée en sodium », « le produit A a une teneur élevée en sodium et le produit B a une faible teneur en sodium ", " le produit A a une faible teneur en sodium et le produit $B$ a une teneur modérée en sodium » et « le produit $\mathrm{A}$ a une teneur modérée en sodium et le produit B a une faible teneur en sodium ».

Manipulation mathématique de I'information nutritionnelle dans les tVN

Nous avons utilisé deux tâches pour examiner la capacité des participants à effectuer la manipulation mathématique de l'information nutritionnelle figurant dans le tVN. Nous avons d'abord posé la question suivante aux participants : « Si vous consommiez la moitié de cette boîte, quel est le \% VQ des lipides totaux que vous consommeriez? " Nous leur avons ensuite demandé : «Combien de portions devriez-vous manger pour combler vos besoins en fibres d'une journée? »

\section{Analyse des données}

Nous avons utilisé des tests du chi carré pour déterminer s'il existait des différences liées aux caractéristiques des participants soumis aux différentes conditions associées au tVN et des différences liées à la proportion de participants ayant répondu correctement aux questions de l'enquête pour chacune des sept tâches liées à ces conditions. Nous avons ensuite élaboré des modèles de régression de Poisson distincts à l'aide des scores combinés obtenus pour les tâches associées aux trois résultats, soit interpréter (2 éléments), comparer (3 éléments) et manipuler mathématiquement (2 éléments) l'information, afin de déterminer le nombre de réponses correctes pour chaque condition expérimentale par rapport à la condition témoin. Nous avons examiné les associations entre les covariables sélectionnées (niveau de scolarité, origine ethnique, situation vis-à-vis de l'emploi, région, objectif relatif au poids, responsabilité de l'achat des aliments et de la préparation des repas, connaissance des recommandations relatives aux calories et qualité perçue de l'alimentation) et chacun des trois résultats à l'aide de modèles incluant l'effet principal (condition), et nous avons ajusté les résultats selon l'âge et le sexe. Les covariables ayant une valeur $p$ inférieure à 0,2 ou modifiant le coefficient bêta de l'effet principal par plus de $20 \%$ ont été incluses dans le modèle final. Les analyses ont été effectuées au moyen du logiciel SAS 9.3 (Cary, Caroline du Nord, États-Unis).

\section{Résultats}

Les caractéristiques des participants sont présentées dans le tableau 1. Aucune différence significative (associée à $p<0,05$ ) n’a été observée entre les conditions expérimentales associées au tVN en ce qui concerne les caractéristiques sociodémographiques et les comportements à l'égard de la nutrition, à l'exception de la connaissance des recommandations relatives aux calories, ce qui témoigne d'une répartition aléatoire adéquate.

\section{Interprétation de l'information sur le \% VQ dans les $t V N$}

Dans les deux premières tâches d'interprétation, la capacité à interpréter correctement l'information sur la teneur en sodium des deux produits a été améliorée de façon significative dans quatre conditions dans lesquelles le tVN était modifié (ajout des descripteurs «faible/moyen/élevé », portion de référence normalisée + ajout des descripteurs « faible/moyen/élevé », ajout d'un code de couleurs au \% VQ, portion de référence normalisée + ajout d'un code de couleurs au \% VQ; $p<0,005$ dans tous les cas) comparativement à la condition témoin (tableau 2). Les participants étaient plus nombreux à interpréter correctement l'information sur les lipides totaux dans l'ensemble des cinq conditions expérimentales où le tVN était modifié par rapport aux participants de la condition témoin ( $p \leq 0,001$ dans tous les cas; tableau 2).

Comme l'illustre le tableau 3, le modèle de régression de Poisson modifié et ajusté indique que la capacité à interpréter correctement l'information a été améliorée de façon significative dans les conditions incluant l'ajout des descripteurs « faible/ 


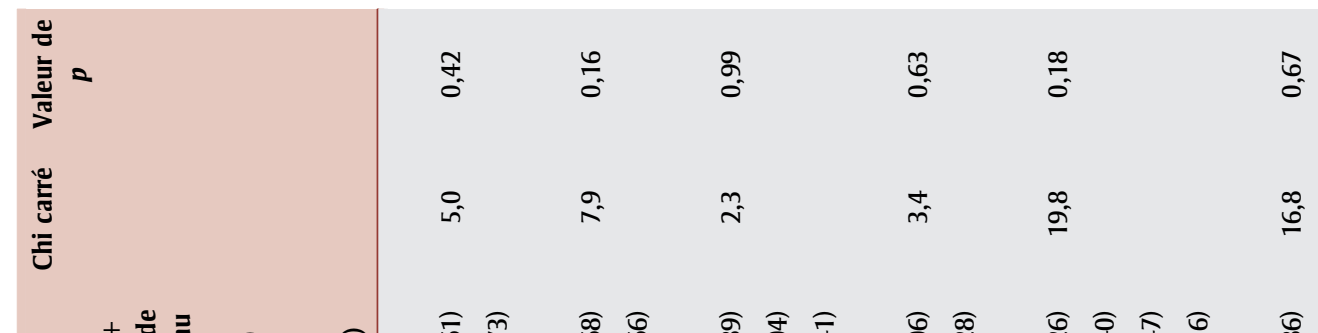

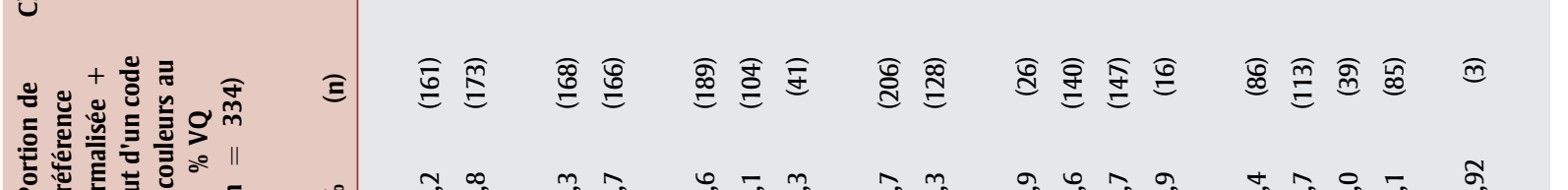

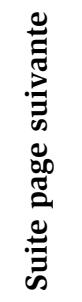

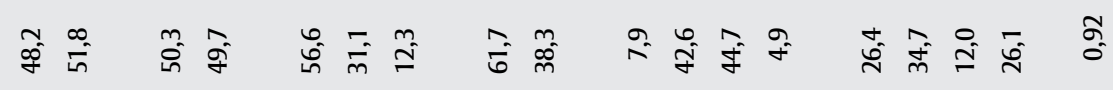

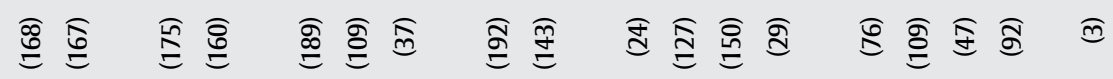

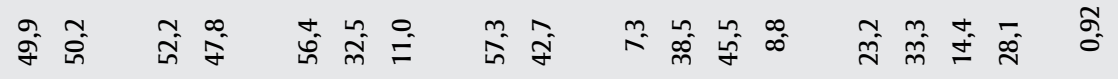

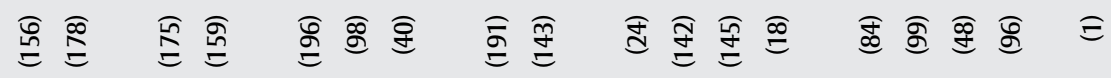

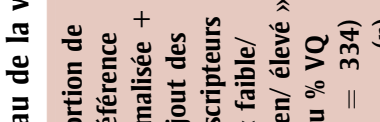

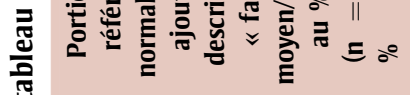

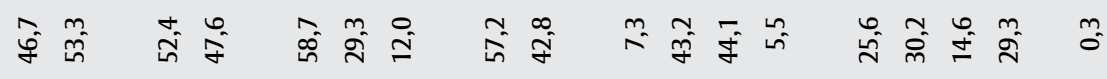

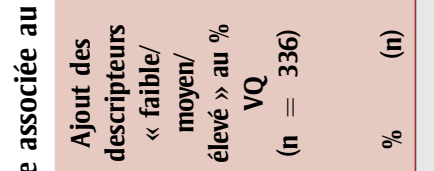

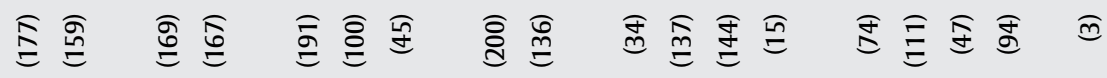

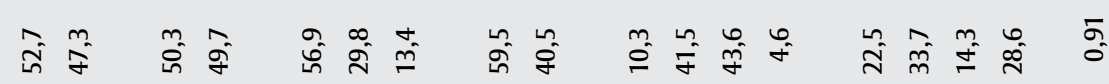
离产 હ

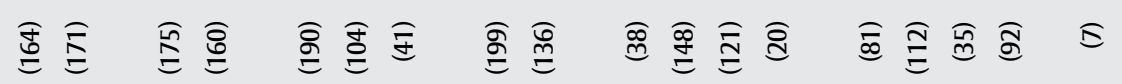

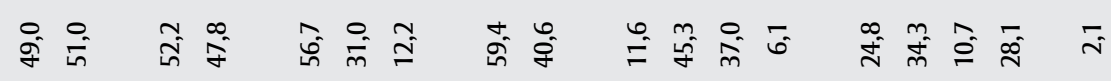

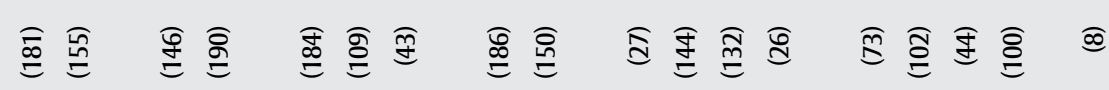

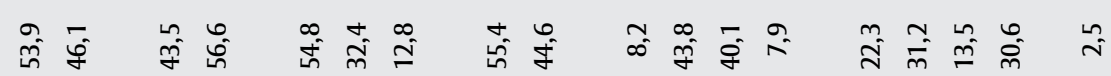

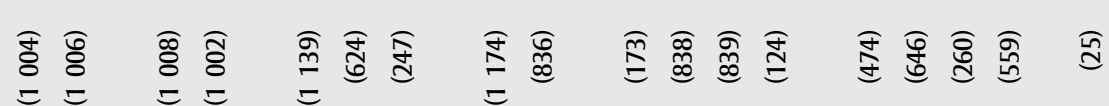

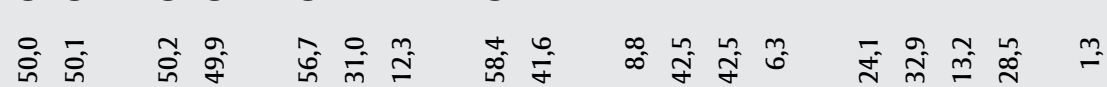
เก เิ
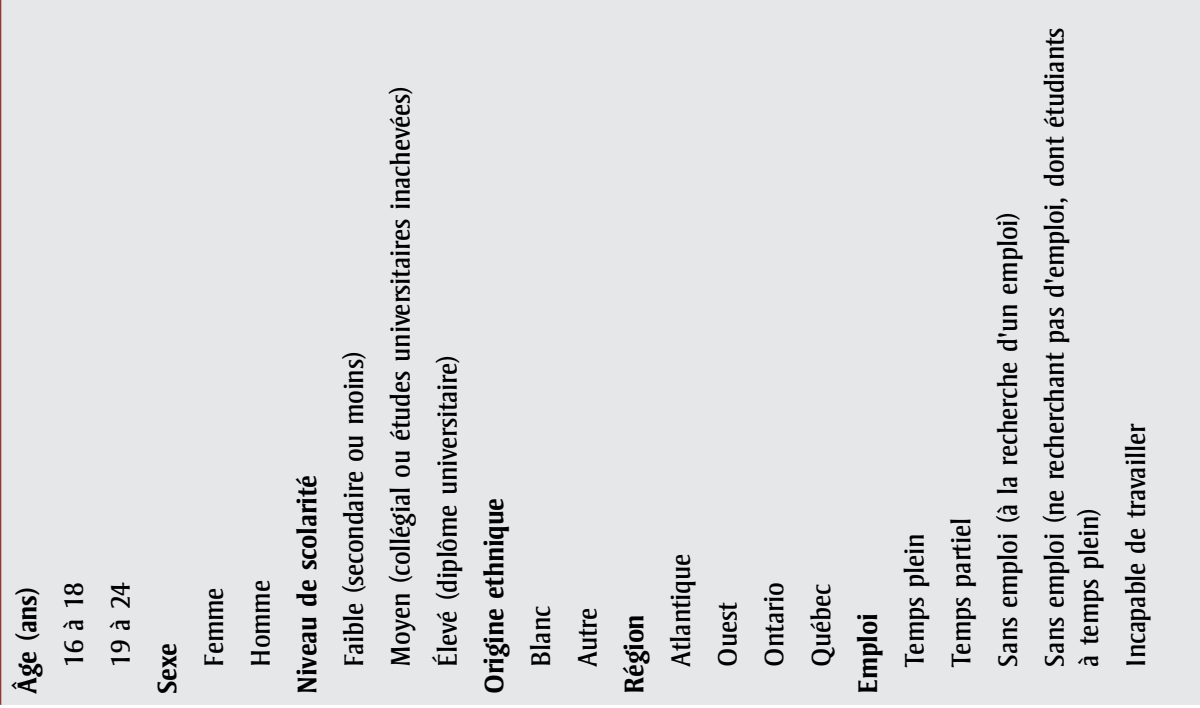


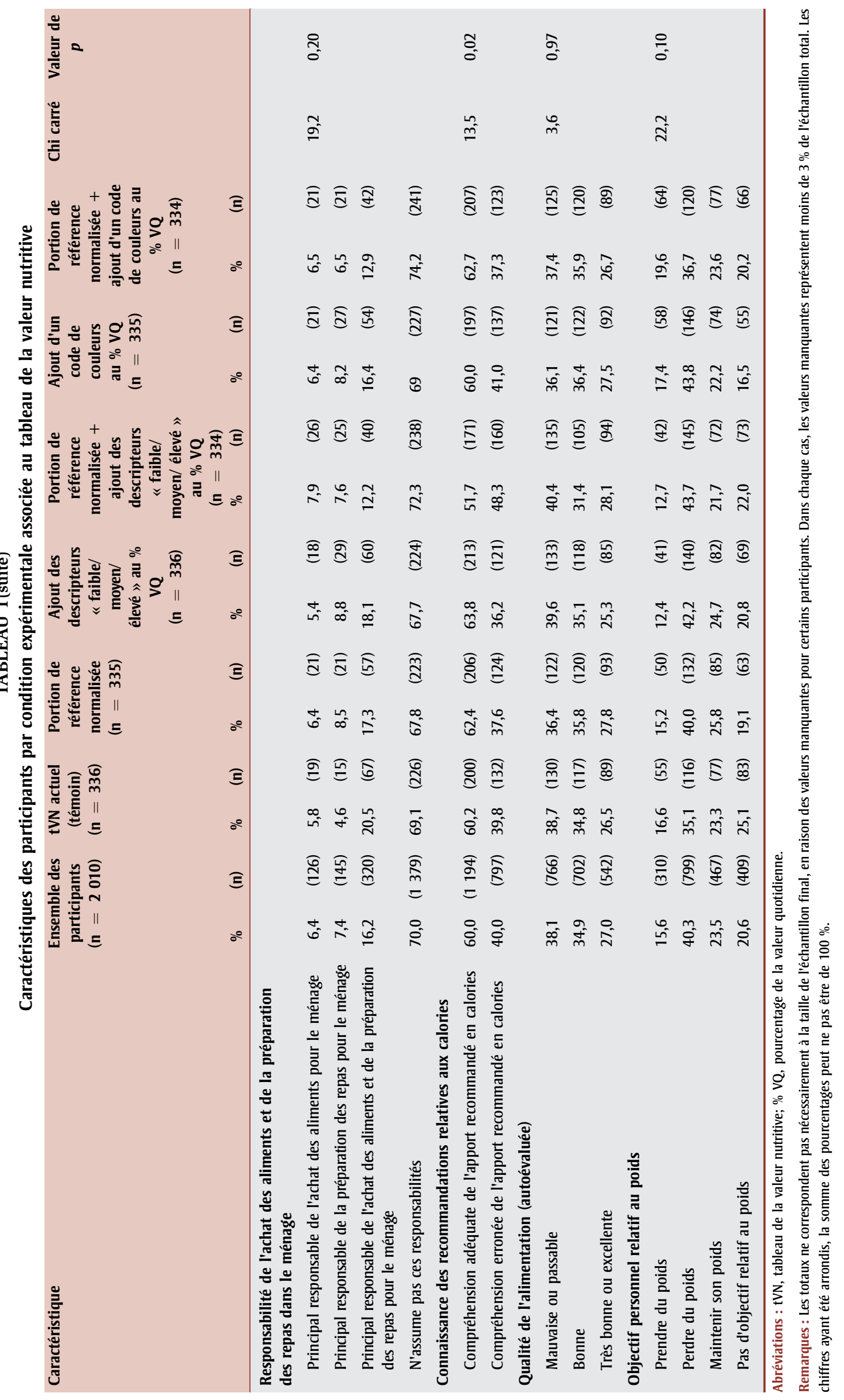




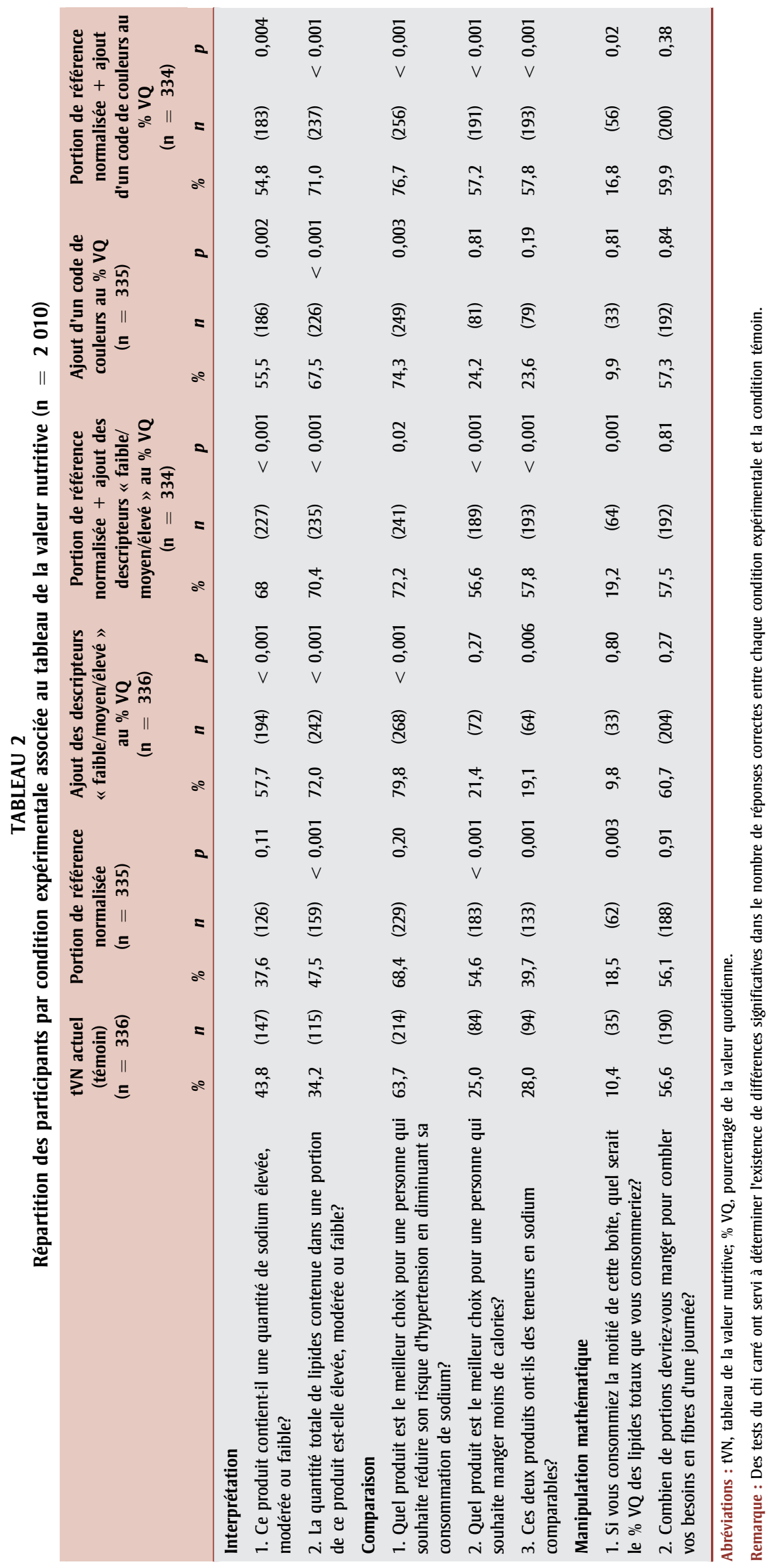


TABLEAU 3

Résultats des modèles de Poisson modifiés évaluant la capacité des participants à interpréter, à comparer et à manipuler, sur le plan mathématique, l'information figurant dans les tableaux de la valeur nutritive associés à chacune des conditions expérimentales par rapport au tableau de la valeur nutritive actuel (condition témoin)

\begin{tabular}{lccccc} 
& $\begin{array}{c}\text { Portion de référence } \\
\text { normalisée }\end{array}$ & $\begin{array}{c}\text { Ajout des descripteurs } \\
\text { « faible/moyen/élevé » } \\
\text { au \% VQ }\end{array}$ & $\begin{array}{c}\text { Portion de référence } \\
\text { normalisée + ajout des } \\
\text { descripteurs « faible/moyen/ } \\
\text { élevé » au \% VQ } \\
\text { RR (IC à } 95 \%)\end{array}$ & $\begin{array}{c}\text { Ajout d'un code } \\
\text { de couleurs } \\
\text { au \% VQ }\end{array}$ & $\begin{array}{c}\text { Portion de référence } \\
\text { normalisée }+ \text { ajout } \\
\text { d'un code de couleurs } \\
\text { au \% VQ }\end{array}$ \\
\hline RR $($ IC à $95 \%)$ & RR (IC à $95 \%)$ & RR (IC à $95 \%)$ & (IC à $95 \%)$ \\
\hline Interprétation $^{\text {a }}$ & $1,11(0,97$ à 1,27$)$ & $1,67(1,48$ à 1,89$)$ & $1,80(1,60$ à 2,03$)$ & $1,61(1,42$ à 1,82$)$ & $1,63(1,44$ à 1,84$)$ \\
Comparaison $^{b}$ & $1,41(1,24$ à 1,59$)$ & $1,03(0,92$ à 1,15$)$ & $1,60(1,53$ à 1,80$)$ & $1,02(0,91$ à 1,15$)$ & $1,64(1,46$ à 1,83$)$ \\
Manipulation $^{c}$ & $1,14(0,99$ à 1,31$)$ & $1,04(0,91$ à 1,19$)$ & $1,19(1,04$ à 1,37$)$ & $1,03(0,90$ à 1,18$)$ & $1,13(0,99$ à 1,29$)$ \\
\hline
\end{tabular}

Abréviations : \% VQ, pourcentage de la valeur quotidienne; RR, ratio relatif; IC à $95 \%$, intervalle de confiance à $95 \%$.

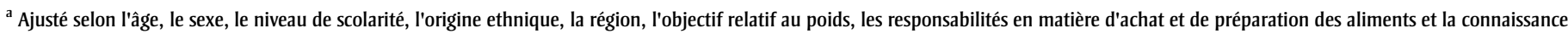
des recommandations relatives aux calories.

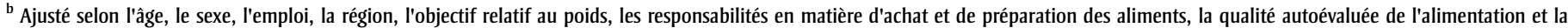
connaissance des recommandations relatives aux calories.

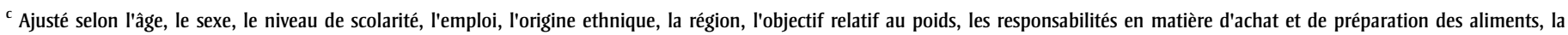
qualité autoévaluée de l'alimentation et la connaissance des recommandations relatives aux calories.

moyen/élevé » $(\mathrm{RR}=1,67$; IC à $95 \%: 1,48$ à 1,89), la portion de référence normalisée + l'ajout des descripteurs « faible/moyen/ élevé » (RR = 1,80; IC à $95 \%: 1,60$ à 2,03), l'ajout d'un code de couleurs au \% VQ $(\mathrm{RR}=1,61$; IC à $95 \%: 1,42$ à 1,82$)$ et la portion de référence normalisée + l'ajout d'un code de couleurs au \% VQ (RR = 1,63; IC à $95 \%: 1,44$ à 1,84). En revanche, la condition incluant la portion de référence normalisée n’a pas amélioré de façon significative la capacité des participants à interpréter correctement l'information figurant dans le $\operatorname{tVN}(p=0,14$; tableau 3).

\section{Comparaison de l'information figurant dans les deux tVN}

Pour la première des trois tâches de comparaison, par rapport à la condition témoin, un nombre significativement supérieur de participants ont comparé correctement les deux tVN et déterminé le produit le plus faible en sodium dans toutes les cas où le tVN était modifié, à l'exception de la condition incluant uniquement la portion de référence normalisée ( $p \leq 0,02$ dans tous les cas; tableau 2).

En ce qui concerne la deuxième tâche de comparaison, les participants ont déterminé avec exactitude, dans une proportion significativement supérieure, le produit contenant le moins de calories dans les conditions du tVN incluant la portion de référence normalisée $(p<0,001)$, la portion de référence normalisée + l'ajout des descripteurs « faible/moyen/élevé » $(p<0,001)$ et la portion de référence normalisée + l'ajout d'un code de couleurs au \% VQ $(p<0,001)$.

Enfin, pour la troisième tâche de comparaison, par rapport à la condition témoin, un nombre significativement supérieur de participants ont comparé correctement la teneur en sodium des deux produits dans trois conditions relatives au tVN, soit lorsque les tVN incluaient la portion de référence normalisée $(p=0,001)$, la portion de référence normalisée + l'ajout des descripteurs «faible/moyen/élevé » au \% VQ $(p<0,001)$ et la portion de référence normalisée + l'ajout d'un code de couleurs au \% VQ $(p<0,001)$. Les participants ont été nettement moins nombreux à comparer avec exactitude l'information relative au sodium lorsque les descripteurs " faible/ moyen/élevé » étaient indiqués $(p=0,006)$.

Selon le modèle de régression de Poisson modifié et ajusté, la capacité des participants à comparer correctement les deux tVN a été améliorée de façon significative dans les conditions où le $\mathrm{tVN}$ incluait la portion de référence normalisée seule $(\mathrm{RR}=1,41$; IC à $95 \%$ : 1,24 à 1,59) ou combinée aux descripteurs « faible/moyen/élevé » $(R R=1,60$; IC à $95 \%: 1,53$ à 1,80 ) ou à un code de couleurs $(\mathrm{RR}=1,64$; IC à $95 \%: 1,46$ à 1,83), comparativement aux participants de la condition témoin (tableau 3).

\section{Manipulation mathématique de I'information nutritionnelle dans les tVN}

Globalement, la capacité des participants à manipuler, du point de vue mathématique, l'information relative aux lipides totaux était faible, ne dépassant pas $20 \%$ dans l'ensemble des conditions. Cependant, l'exactitude était supérieure de façon significative dans trois conditions associées au tVN (portion de référence normalisée, portion de référence normalisée + descripteurs «faible/moyen/élevé », portion de référence normalisée + ajout d'un code de couleurs au \% VQ; $p<0,05$ dans tous les cas), comparativement à la condition témoin (tableau 2). Plus de la moitié des participants ont pu manipuler correctement, du point de vue mathématique, l'information relative aux fibres dans l'ensemble des cinq conditions expérimentales, de même que dans la condition témoin. Toutefois, globalement, aucune condition n'a eu d'effet perceptible ( $p=0,79$ ).

Les résultats du modèle de régression de Poisson modifié et ajusté indiquent que les tVN qui comprenaient une portion de référence normalisée et des descripteurs « faible/moyen/élevé » amélioraient de façon modeste mais significative la capacité des participants à manipuler correctement l'information nutritionnelle du point de vue mathématique par rapport au tVN témoin $(\mathrm{RR}=1,19$; IC à $95 \%: 1,04$ à 1,37; tableau 3). Aucune autre modalité 
d'étiquetage n'a permis d'améliorer de façon significative la capacité des participants à manipuler mathématiquement l'information fournie dans les tVN.

\section{Analyse}

Cette étude est l'une des premières études évaluées par les pairs au Canada à se pencher sur les effets de modifications apportées au tVN sur le plan de la compréhension et de l'utilisation de l'information nutritionnelle par les jeunes. Il s'agit en outre de l'une des premières études empiriques à l'échelle internationale menées auprès des jeunes ${ }^{19,21}$. Nos résultats révèlent que la normalisation des portions de référence et l'inclusion de descripteurs simples ou de code de couleurs permettant d'interpréter le \% VQ dans le tVN augmentent la capacité des adolescents et des jeunes adultes à interpréter, à comparer et à manipuler, d'un point de vue mathématique, l'information nutritionnelle.

L'emploi de portions de référence normalisées dans les tVN a permis d'améliorer grandement la capacité des jeunes à comparer deux aliments similaires. Des données probantes antérieures semblent indiquer que l'absence d'uniformité entre les portions qui figurent dans les tVN d'un produit à l'autre peut orienter les perceptions et les intentions d'achat en faveur du produit dont la portion de référence est la plus petite, indépendamment de sa supériorité du point de vue nutritionnel ${ }^{31}$. Le fait d'exiger des fabricants qu'ils utilisent des portions de référence normalisées pour des produits similaires pourrait être une stratégie prometteuse, qui faciliterait la compréhension et la bonne utilisation de l'étiquetage nutritionnel des aliments.

L'ajout de descripteurs ou de codes de couleurs à côté des calories et de la teneur en nutriments dans les tVN a eu un effet déterminant sur la capacité des jeunes à interpréter correctement l'information relative au \% VQ. Ces observations rejoignent les résultats d'études et de rapports d'experts qui se sont intéressés aux systèmes d'étiquetage des aliments sur les emballages ${ }^{32}$. Les descripteurs ou les codes de couleurs sont des outils d'interprétation susceptibles d'aider les consommateurs à décoder l'information nutritionnelle complexe présentée sous forme numérique, en limitant les connaissances nécessaires relatives à l'alimentation, les efforts cognitifs et le temps d'analyse requis. Des experts ont souligné à quel point il était important de trouver des stratégies permettant de communiquer une information nutritionnelle complexe sous une forme intelligible pour les consommateurs, plutôt que de recourir exclusivement aux données numériques (kilocalories, grammes, milligrammes et pourcentages) ${ }^{33}$. Notre étude ayant mis à l'essai des outils d'interprétation portant exclusivement sur les teneurs en calories et en nutriments négatifs, d'autres recherches sont à mener afin de définir les modalités d'application de cette approche aux nutriments positifs.

Conformément aux résultats d'une autre recherche $^{34}$, bon nombre de participants ont eu de la difficulté à manipuler l'information nutritionnelle afin de calculer la teneur en nutriments de plusieurs portions, en particulier lorsque la tâche exigeait des opérations mathématiques complexes et la compréhension de l'information sur le $\%$ VQ. L'inclusion de portions de référence normalisées et de descripteurs simples dans les tVN a permis d'améliorer la capacité des participants à manipuler mathématiquement l'information nutritionnelle, en particulier au cours de la tâche impliquant d'additionner plusieurs portions d'un produit et d'évaluer le \% VQ correspondant à la somme. Cependant, cet effet a été modeste, et la majorité des participants ont tout de même été incapables de manipuler et d'utiliser correctement l'information numérique présentée sur les étiquettes. L'une des explications à la difficulté générale que pose la manipulation de l'information nutritionnelle pourrait notamment provenir du fait que ce type de tâche requiert, outre des connaissances en nutrition et des habiletés mathématiques, un temps, une motivation et des efforts considérables. Afin de faciliter et d'accélérer la consultation de l'information nutritionnelle, les auteurs d'autres études ont proposé l'ajout d'une deuxième colonne au tVN où seraient indiqués les nutriments et les calories contenus dans l'emballage au complet $^{35}$. Cette modification pourrait aider les consommateurs à évaluer le profil nutritionnel des produits qui contiennent plusieurs portions. Cependant, Roberto et Khandpur $^{36}$ ont fait remarquer que l'ajout de renseignements supplémentaires pourrait rendre les étiquettes plus complexes. D'autres recherches sont à mener pour comparer les étiquettes à deux colonnes et à une colonne aux formats de présentation simplifiés incluant moins d'information, dont une indication dans le tVN du nombre total de portions par emballage.

\section{Points forts et limites}

Notre étude comporte plusieurs limites. Tout d'abord, nous n'avons pas utilisé de technique d'échantillonnage probabiliste pour sélectionner un échantillon représentatif de jeunes Canadiens. L'échantillon était plutôt transversal, fournissant un groupe hétérogène de participants à l'échelle du Canada en vue de leur répartition aléatoire dans les diverses conditions associées au tVN. Des recherches antérieures ont montré que des niveaux de revenus et d'études supérieurs étaient généralement associés à de meilleures performances dans les tâches relatives à l'étiquetage nutritionnel ${ }^{19}$. Or plus de $76 \%$ des jeunes adultes (de 19 à 24 ans) de notre échantillon avaient un niveau de scolarité supérieur aux études secondaires. Compte tenu de la piètre performance aux tâches dans notre échantillon où le niveau de scolarité était très élevé, on peut supposer que les consommateurs dans leur ensemble seraient encore moins aptes à comprendre et à utiliser l'information relative aux portions de référence et au $\% \mathrm{VQ}$. Bien que nos constatations soient susceptibles de s'appliquer aux autres Canadiens du même groupe d'âge, d'autres recherches sont requises afin de vérifier si les résultats seraient similaires pour certains sousgroupes de jeunes non visés par cette étude, notamment les locuteurs d'autres langues que l'anglais et les personnes peu susceptibles d'être recrutées ou de participer à une recherche en ligne. Afin de mieux simuler une situation réelle, les tVN ont été affichés sur deux boîtes de craquelins de marques hypothétiques. Les craquelins conviennent à la réalisation d'essais sur différents formats de présentation de l'information nutritionnelle, étant donné les variations nutritives qu'ils présentent, et aussi parce que du point de vue nutritif, c'est un choix considéré ni comme très bon ni comme très mauvais pour la santé. De nombreuses études utilisent une seule denrée préemballée dans le cadre d'essais 
sur différents formats de présentation de l'information nutritionnelle et appliquent les résultats de façon généralisée à d'autres produits alimentaires ${ }^{16,36-38}$. ll est cependant recommandé de reproduire cette étude en utilisant d'autres produits alimentaires et d'autres catégories d'aliments, puisque les résultats pourraient varier. Enfin, même si notre étude a utilisé une méthode classique d'évaluation d'un matériel et de concepts de communication avant leur mise en œuvre, aucune étude expérimentale menée en ligne ne peut reproduire une véritable expérience d'achat. Les recherches futures devraient viser à évaluer l'efficacité des modifications apportées aux tVN quant aux choix d'aliments et aux comportements alimentaires dans des conditions fidèles à la réalité.

\section{Conclusion}

Les spécialistes du milieu universitaire comme ceux des organismes de santé ont recommandé des améliorations à l'étiquetage nutritionnel, dont la normalisation des portions de référence et l'ajout de mentions facilitant l'interprétation du \% VQ, changements que les consommateurs souhaitent également ${ }^{22,39,40}$. D'éventuelles modifications de l'étiquetage nutritionnel sont en cours d'examen au Canada, notamment la normalisation des portions de référence au sein des catégories de produits similaires et l'ajout d'explications sur ce qu'est un faible et un grand $\% \mathrm{VQ}^{22}$. Notre recherche donne à penser que ces deux modifications pourraient aider les jeunes Canadiens à bien interpréter l'information qui figure dans le tVN lorsqu'ils choisissent des aliments, comparent des produits similaires et manipulent sur le plan mathématique des données numériques pour déterminer le contenu nutritionnel de plusieurs portions d'un produit.

Nos constatations fournissent des données probantes préliminaires qui montrent que l'on peut aider le consommateur à comprendre et à utiliser l'information nutritionnelle en apportant certaines modifications au tVN. Des recherches supplémentaires sont à mener pour préciser si les modifications apportées au tVN favorisent un choix alimentaire éclairé pour d'autres catégories d'aliments et chez les adultes des autres tranches d'âge au Canada.

\section{Remerciements}

Cette étude a été financée par la Fondation canadienne de la recherche en diététique.

\section{Références}

1. Global Burden of Disease Study 2010. Country Profiles - GBD Profile Canada [Internet]. Washington (DC) : Institute for Health Metrics and Evaluation; [consultation le 8 juillet 2015]. PDF téléchargeable à partir du lien : http://www.healthdata.org/sites/default/ files/files/country_profiles/GBD/ihme_gbd_ country_report_canada.pdf

2. Hooper L, Martin N, Abdelhamid A, Davey Smith G. Reduction in saturated fat intake for cardiovascular disease. Cochrane Database Syst Rev. 2015;6:CD011737 DOI: 10.1002/ 14651858.CD011737.

3. Strazzulo P, D’Elia L, Kandala NB, Cappuccio FP. Salt intake, stroke, and cardiovascular disease: meta-analysis of prospective studies. BMJ. 2009;339:b4567.

4. Abdullaha A, Peetersa A, de Courtena M, Stoelwinder J. The magnitude of association between overweight and obesity and the risk of diabetes: a meta-analysis of prospective cohort studies. Diabetes Res Clin Pract. 2010;89(3):309-319.

5. Amed S, Dean HJ, Panagiotopoulos C et collab. Type 2 Diabetes, medication-induced diabetes, and monogenic diabetes in Canadian children: a prospective national surveillance study. Diabetes Care. 2010;33(4):786-791.

6. Shields M. L'embonpoint et l'obésité chez les enfants et les adolescents. Rapports sur la santé. 2006;17(3):27-42. PDF téléchargeable à partir du lien : http://www.statcan.gc.ca/ pub/82-003-x/2005003/article/9277-fra.pdf

7. Agence de la santé publique du Canada. Rapport de l'administrateur en chef de santé publique sur l'état de la santé publique au Canada 2011 - Jeunes et jeunes adultes - En période de transition. Ottawa (Ont.) : Agence de la santé publique du Canada; 2011. Consultable en ligne à la page : http://www.phac-aspc.gc.ca/ cphorsphc-respcacsp/2011/index-fra.php

8. Ribiero RC, Coutinho M, Bramorski MA, Giuliano IC, Pavan J. Association of the waist-to-height ratio with cardiovascular risk factors in children and adolescents: the Three Cities Heart Study. Int J Prev Med. 2010;1:39-49.
9. Nelson MC, Story M, Larson NI, NeumarkSztainer D, Lytle LA. Emerging adulthood and college-aged youth: an overlooked age for weight-related behavior change. Obesity. 2008;16(10):2205-2211.

10. Larson NI, Story M, Eisenberg ME, NeumarkSztainer D. Food preparation and purchasing roles among adolescents: associations with sociodemographic characteristics and diet quality. J Am Diet Assoc. 2006;106(2)211-218.

11. Dunn JE, Liu K, Greenland P, Hilner JE, Jacobs DR Jr. Seven-year tracking of dietary factors in young adults: the CARDIA study. Am J Prev Med. 2000;18(1):38-45.

12. Santé Canada. Règlement modifiant le Règlement sur les aliments et drogues (étiquetage nutritionnel, allégations relatives à la teneur nutritive et allégations relatives à la santé). Gazette du Canada, Partie 2. $1^{\mathrm{er}}$ janvier 2003;137(1):154. PDF téléchargeable à partir du lien : http://publications.gc.ca/ gazette/archives/p2/2003/2003-01-01/pdf/g213701.pdf

13. Goodman S, Hammond D, Pillo-Blocka F, Glanville T, Jenkins R. Use of nutritional information in Canada: national trends between 2004 and 2008. J Nutr Educ Behav. 2011;43:356-365.

14. Emrich TE, Qi Y, Mendoza JE, Lou W, Cohen JE, L'abbe MR. Consumer perceptions of the Nutrition Facts table and front-of-pack nutrition rating systems. Appl Physiol Nutr Metab. 2014;39(4):417-424.

15. Cowburn G, Stockley L. Consumer understanding and use of nutrition labelling: a systematic review. Public Health Nutr. 2005;8(1):21-28.

16. The Strategic Counsel. Canadians' understanding and use of the nutrition facts table: baseline national survey results. 2011; POR 031-10(HCPOR-10-06).

17. Agence canadienne d'inspection des aliments. Information contenue dans le tableau de la valeur nutritive. Renseignements obligatoires et portion déterminée [Internet]. Ottawa (Ont.) : Agence canadienne d'inspection des aliments; [modification le 10 avril 2015; consultation le 15 juillet 2015]. Consultable en ligne à la page : http://www.inspection.gc.ca/ aliments/etiquetage/l-etiquetage-des-alimentspour-l-industrie/etiquetage-nutritionnel/information-contenue-dans-le-tableau-de-la-valeur-/ fra/1389198568400/1389198597278?chap = 1 
18. Gouvernement du Canada. Étiquetage des aliments : pourcentage de la valeur quotidienne [Internet]. Ottawa (Ont.) : Gouvernement du Canada; [modification le 29 mai 2015; consultation le 15 juillet 2015]. Consultable en ligne à la page : http://www.canadiensensante. gc.ca/eating-nutrition/label-etiquetage/dailyvalue-valeur-quotidienne-fra.php

19. Campos S, Doxey J, Hammond D. Nutrition labels on pre-packaged foods: a systematic review. Public Health Nutr. 2011;14(8) 1496-1506.

20. Wartella EA, Lichtenstein AH, Boon CS Editors. Committee on Examination of Frontof-Package Nutrition Ratings Systems and Symbols; Institute of Medicine Institute of Medicine (IOM). Front-of-Package Nutrition Rating Systems and Symbols. Phase 1 Report. Washington (DC) : The National Academies Press; 2010.

21. Wojcicki JM, Heyman MB. Adolescent nutritional awareness and use of food labels: results from the National Nutrition Health and Examination Survey. BMC Pediatr. 2012;12:55.

22. Santé Canada. Modifications proposées de Santé Canada aux exigences de présentation de l'information nutritionnelle et d'autres renseignements sur l'étiquette des aliments [Internet]. Ottawa (Ont.) : Santé Canada; 14 juillet 2014 [consultation le 15 juillet 2015]. Consultable en ligne à la page : http://www.hc-sc.gc.ca/fn-an/consult/2014format-requirements-exigences-presentation/ document-consultation-fra.php\#a1

23. Balasubramanian SK, Cole C. Consumers' search and use of nutrition information: the challenge and promise of the Nutrition Labeling and Education Act. J Marketing. 2002;66(3):112-127.

24. Santé Canada. Le sodium au Canada : l'apport recommandé en sodium [Internet]. Ottawa (Ont.) : Santé Canada; 8 juin 2012 [consultation le 13 juillet 2015]. Consultable en ligne à la page : http://www.hc-sc.gc.ca/ fn-an/nutrition/sodium/index-fra.php\#a2

25. Centers for Disease Control and Prevention. 2011-2012 National Health and Nutrition Examination Survey (NHANES) - Weight history - WHQ: Target group: SPs $16+$ [Internet]. Atlanta (Ga.) : NHANES; 2011. PDF téléchargeable à partir du lien : http:// www.cdc.gov/nchs/data/nhanes/nhanes_11_ 12/whq.pdf
26. Bleich SN, Pollack KM. The publics' understanding of daily caloric recommendations and their perceptions of calorie posting in chain restaurants. BMC Public Health. 2010;10:121.

27. Elbel B. Consumer estimation of recommended and actual calories at fast food restaurants. Obesity. 2011;19:1971-1978.

28. Krukowski RA, Harvey-Berino J, Kolodinsky J, Narsana RT, Desisto TP. Consumers may not use or understand calorie labeling in restaurants. J Am Diet Assoc. 2006;106:917-920.

29. Santé Canada. Aliments et nutrition : besoins énergétiques estimatifs [Internet]. Ottawa (Ont.) : Santé Canada; [modification le 20 mars 2014; consultation le 13 juillet 2015]. Consultable en ligne à la page : http://www. hc-sc.gc.ca/fn-an/food-guide-aliment/basicsbase/1_1_1-fra.php

30. Mackison D, Wrieden WL, Anderson AS. Validity and reliability testing developed to assess consumers' use, understanding, and perceptions of food labels. Eur J Clin Nutr. 2010;64(2):210-217.

31. Mohr GS, Lichtenstein DR, Janiszewski C. The effect of marketer-suggested serving size on consumer responses: the unintended consequences of consumer attention to calorie information. J Marketing. 2012;76 (59):75.

32. Wartella EA, Lichtenstein AH, Yaktine A, Nathan R (dir.), Committee on Examination of Front-of-Package Nutrition Rating Systems and Symbols (Phase II). Front-ofpackage nutrition rating systems and symbols: promoting healthier choices. Washington (DC) : The National Academies Press; 2011.

33. Van Herpen E, Hieke S, van Trijp H.C.M. Inferring product healthfulness from nutrition labelling. The influence of reference points. Appetite. 2014;72:138-149.

34. Rothman RL, Housam R, Weiss H et collab. Patient understanding of food labels: the role of literacy and numeracy. Am J Prev Med. 2006;31(5):391-398.

35. Antonuk B, Block LG. The effect of single serving versus entire package nutritional information on consumption norms and actual consumption of a snack food. J Nutr Educ Behav. 2006;38(6):365-370.
36. Hieke S, Wilczynski P. Colour me in - an empirical study on consumer responses to the traffic light signposting system in nutrition labelling. Public Health Nutr. 2013; 15(5):773-82.

37. Andrews J, Burton S, Kees J. Is simpler always better? Consumer evaluations of front-of-package nutrition symbols. J Public Policy \& Marketing. 2011;30(2):175-90.

38. Hamlin R, McNeill L, Moore V. The impact of front-of-pack nutrition labels on consumer product evaluation and choice: an experimental study. Public Health Nutr. 18(12):2126-34.

39. Roberto CA, Khandpur N. Improving the design of nutrition labels to promote healthier food choices and reasonable portion sizes. Int J Obes (Lond). 2014;38 Suppl 1:S25-S33.

40. Silverglade B, Ringel Heller I. Food labeling chaos: the case for reform [Internet]. Washington (DC) : Center for Science in the Public Interest; 2010 [consultation le 15 juillet 2015]. PDF téléchargeable à partir du lien : http://www.cspinet.org/new/pdf/food_ labeling_chaos_report.pdf 